\section{Depression und \\ Bildgebung}

Christian Vollmert und Dieter F. Braus

Neurolmage Nord - Psychiatrie und Klinik für Psychiatrie und Psychotherapie

Universitätsklinikum Hamburg-Eppendorf

psychoneuro 2005; 31 (4): 204-211

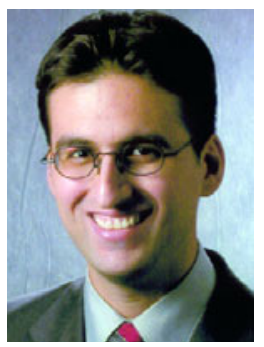

Christian Vollmert
Das Etablieren pathogenetischer Netzwerkmodelle für depressive Störungen wurde durch die moderne Bildgebung nachhaltig gefördert. Distinkte morphologische Befunde weisen bei depressiven Störungen auf eine relativ abgrenzbare Verteilung von Auffälligkeiten im Bereich emotional relevanter Regelkreise des Gehirns hin. Die funktionelle Bildgebung konzentriert sich auf neuronale Störungen im Bereich des Frontalhirns, des limbischen Systems mit der Hip pokampus-Amygdala-Formation sowie der Basalganglien. Affektmodulation erfordert ein geordnetes Zusammenspiel ventral-limbischer mit dorsal-neokortikalen Hirnregionen, die bei depressiven Störungen in Dysbalance geraten, wobei dem rostralen anterioren Cingulum möglicherweise eine Schlüsselstellung dabei zukommt. Aufbauend auf diesem Modell zielt die bildgebende Forschung zukünftig auf die Identifikation valider neurofunktioneller Subgruppen unter Einbezug von Vulnerabilitätsgenen ab, auch um effizientere Behandlungsstrategien entwickeln zu können.

$\mathrm{M}$ it Hilfe der funktionellen Kernspintomographie (fMRT) und der PositronenEmissions-Tomographie (PET) können einerseits Einzelkomponenten neuronaler Module in ihrer Interaktion auf der Systemebene untersucht werden, mit der Diffusions-TensorBildgebung (DTI) und der MR-Spektroskopie (MRS) werden andererseits aber auch Einblicke in die mikrostrukturellen und biochemischen Besonderheiten des lebenden Gehirns möglich. Nachfolgend sollen wesentliche Befunde der letzten Jahre aus diesem Forschungsbereich zu affektiven Störungen zusammengefasst werden, sowie ein daraus folgendes Netzwerkmodell der Depression abgeleitet werden mit möglichen Konsequenzen für DifferentialDiagnostik und -Therapie.

\section{Morphologische Bildgebung (MR-Volumetrie)}

Seit der Einführung der Computertomographie in den 70er-Jahren hat die morphologische Untersuchung des Gehirns bei verschiedenen psychiatrischen Störungen eine lange Tradition. So wurde bei Patienten mit majorer Depression eine verringerte zerebrale Asymmetrie sowie ein verringertes Frontallappenvolumen nachgewiesen. Auf Subgruppenebene wurden eine Vergrößerung der Liquorräume bei Patienten mit psychotischen Symptomen sowie diskrete Veränderungen im frontalen Marklager in Form periventrikulärer und subkortikaler Signalveränderungen (sog. White matter lesions, WML) bei Patienten mit spätem Erkrankungsbeginn berichtet (Abb. 1). Die Kombination von Histomorphologie und verbesserten MRT-Methoden zeigte später einen Zusammenhang dieser MRTVeränderungen - insbesondere im funktionell relevanten frontalen Marklager - mit ischämischen Läsionen auf (29).

Die Befunde weisen auf eine besondere Bedeutung des Frontalhirns hin, das mit limbischen Arealen wie der Hippokampus-Amygdala-Formation und dem rostralen anterioren Cingulum in enger Beziehung steht. Neben struktureller Volumenverminderung in subgenualen präfrontalen Arealen (6) (Abb. 2) sind diese auch zwischenzeitlich in orbitofrontalen Regionen beschrieben, zum Teil auch unabhängig von vaskulären Faktoren zu finden (27). Subkortikal lassen sich außerdem Läsionen der Basalganglien nachweisen, denen nach Ahearn und Mitarbeitern (2) eine prognostische Bedeutung für das Risiko eines Suizidversuchs zukommt.

In Übereinstimmung mit histopathologischen Befunden wurden auch MR-volumetrische Volumenreduktionen bzw. strukturelle Veränderungen des Hippokampus und der Amygdala bei Patienten mit depressiver Störung wiederholt beschrieben (31). In jüngerer Zeit wurde ein Zusammenhang zwischen der Abnahme des Hippokampusvolumens 
mit der langen Variante (1-Allel) des Serotonin-Transporter-Polymorphismus nachgewiesen (9), ein Befund, der jedoch erst der unabhängigen Replikation bedarf.

Die berichteten morphologischen Daten zur depressiven Störung weisen insgesamt auf eine relativ abgrenzbare Verteilung von Auffälligkeiten im Bereich des Frontallappens, subgenual, der Basalganglien sowie der HippokampusAmygdala-Formation hin, also in den emotional relevanten Regelkreisen des Gehirns.

\section{Metabolische Bildgebung (MR-Spektroskopie)}

Mit Hilfe der MR-Spektroskopie (MRS) können verschiedene Metaboliten im Hirngewebe quantifiziert werden. So kann mit der Protonenspektroskopie das N-Acetyl-Aspartat (NAA)-Signal gemessen werden, das Hinweise auf die Integrität und Funktionsfähigkeit der Neuronen liefert. In MR-Spektren können weiterhin Cholin, Kreatin sowie mit speziellen Editierungsverfahren die Neurotransmitter Gamma-Aminobuttersäure (GABA) und Glutamat/Glutamin quantifiziert werden. Dabei wird eine Zunahme des CholinSignals aufgrund seiner hohen Konzentration in Zellmembranlipiden als Hinweis auf einen vermehrten zellulären Membran-Turnover interpretiert. Dieser kommt z.B. bei der Zellproliferation und Synapsenbildung im Zusammenhang mit neuroplastischen Prozessen des Gehirns vor. Das Kreatin-Signal erlaubt wie einige der erfassten Phosphorspektroskopie-Metaboliten (z.B. ATP) Rückschlüsse auf den Zustand des intrazellulären Energiemetabolismus.

Bei depressiven Patienten konnten als Hinweis für eine Funktionsstörung verminderte ATP-Konzentrationen im Frontallappen nachgewiesen werden. Wiederholt wurde weiterhin - wenn auch nicht immer richtungskonsistent - über Auffälligkeiten der Cholin-Konzentration verschiedener Hirnareale berichtet. Dies wird als Hinweis auf einen pathologisch veränderten Membranphospholipid-Metabolismus im Zusammenhang mit gestörter neuronaler Plastizität gedeutet. Die Wirkung von Antidepressiva scheint dabei mit einer Normalisierung des CholinSignals in den Basalganglien einherzugehen (24). Eine entsprechende Cholin-Veränderung im Hippokampus wurde in eigener Untersuchung bei Patienten mit schwerer depressiven Störung im Verlauf einer ElektroKrampf-Therapie-Serie (EKT) beobachtet, wobei ein unverändertes NAA-Signal als Ausbleiben einer strukturellen Schädigung der Neurone durch die EKT interpretiert werden kann (8). Im Langzeitverlauf nimmt dann Cholin wieder ab, während NAA weiter stabil bleibt (22). Dazu passend zeigte sich auch beim Diskontinuationssyndrom mit selektiven Serotonin-Reuptake-Inhibitoren ein Cholinabfall (13). Insgesamt scheint das Cholin-Signal also auch bei affektiven Störungen Veränderungen neuroplastischer Prozesse abzubilden (siehe auch Beitrag von Fuchs und Flügge in diesem Heft).

\section{Funktionelle Untersuchun- gen unter Ruhebedingungen}

Korrespondierend zu den morphologischen und metabolischen Befunden ergaben sich erste Hinweise auf einen subkortikalen und kortikalen Hypometabolismus unter Ruhebedingungen bei älteren depressiven Patienten bereits Anfang der 90er-Jahre. Funktionelle bildgebende Verfahren wie die PositronenEmissions-Tomographie (PET) und die Single-Photon-Emissions-Computer-Tomographie (SPECT) wurden dabei verwandt. In den letzten Jahren wurde mit Verbesserung der Bildgebungstechnik ein zunehmend differenziertes Bild über die bei der depressiven Störung veränderten funktionellen Regelkreise des Gehirns erarbeitet. Die funktionellen Veränderungen konzentrierten sich dabei im Einklang mit den morphologischen Befunden auf die emotional relevanten Regelkreise des Gehirns (limbisches System mit Hippokampus-Amygdala-Formation, Basalganglien und Frontalhirn).

Interessant ist dabei, dass eine beobachtete Hyperaktivität in der Hippokampus-Amygdala-Formation und ventromedialen präfrontalen Arealen mit dem Ausmaß negativen Affekterlebens zusammenhängt, eine mögliche Erklärung für die Neigung depressiver Patienten zu negativen Kognitionen. Unter einer erfolgreichen antidepressiven Medikation lässt sich eine Normalisierung der Ruheaktivität der HippokampusAmygdala-Region erkennen. Weiterhin zeigten einige Studien einen $\mathrm{Zu}$ sammenhang zwischen beobachteter dorsolateraler Hypofrontalität und der Ausprägung depressiver Sympto-

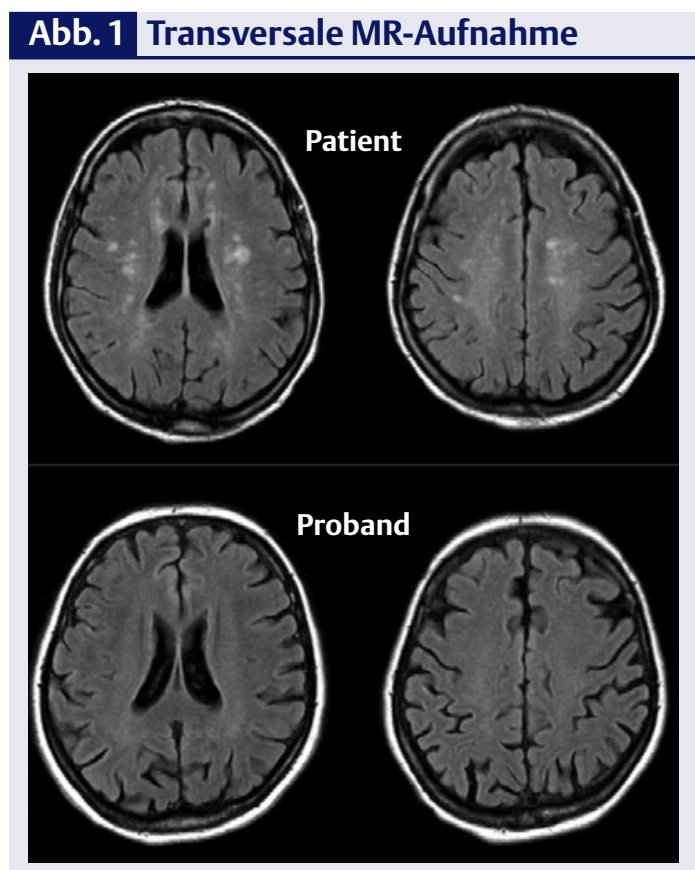

Transversale MR-Aufnahmen (Flair) oben: bei einem älteren Patienten mit einer depressiven Störung mit zahlreichen periventrikulären Whitematter-lesions unten: bei einem altersentsprechenden Kontrollprobanden (nach 32)

\section{Abb. 2 Volumenminderung}

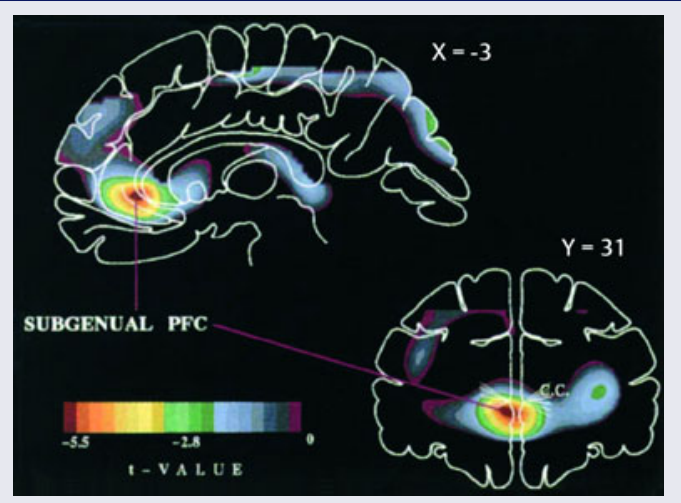

Subgenuale präfrontale Volumenminderung bei affektiven Störungen (nach 6) als erster Hinweis für die besondere Schlüsselstellung dieser Hirnregion für die Affektregulation 


\section{Tab. 1 Ausgewählte funktionelle Befunde unter Ruhebedingungen („resting state“) bei depressiven Störungen}

\begin{tabular}{|c|c|c|c|}
\hline Autoren & Stichprobe & $\begin{array}{l}\text { Untersuchungs- } \\
\text { methode }\end{array}$ & Hauptbefunde \\
\hline \multicolumn{4}{|l|}{ Kognitionsnetzwerk (dorsal) } \\
\hline Bremner et al. (1997) (3) & Patienten $(n=21)$ & FDG-18F-PET & $\begin{array}{l}\text { Verminderter Metabolismus im DLPFK, orbitofrontalen Kortex } \\
\text { und Thalamus bei induziertem depressiven Rückfall }\end{array}$ \\
\hline Kegeles et al. (2003) (14) & $\begin{array}{l}\text { Patienten }(n=19) \\
\text { Gesunde }(n=10)\end{array}$ & FDG-18F-PET & $\begin{array}{l}\text { Verminderter Metabolismus im ventromedialen präfrontalen } \\
\text { Kortex }\end{array}$ \\
\hline \multicolumn{4}{|c|}{ Emotionsnetzwerk (ventral) } \\
\hline Abercrombie et al. (1998) (1) & $\begin{array}{l}\text { Patienten }(n=27) \\
\text { Gesunde }(n=24)\end{array}$ & FDG-18F-PET & $\begin{array}{l}\text { Metabolismus der AMY re. korreliert mit Disposition zu negativen } \\
\text { Emotionen }\end{array}$ \\
\hline Videbech et al. (2002) (30) & $\begin{array}{l}\text { Patienten }(n=42) \\
\text { Gesunde }(n=47)\end{array}$ & $\mathrm{H}_{2}{ }^{15} \mathrm{O}-\mathrm{PET}$ & $\begin{array}{l}\text { Gesteigerter rCBF im HPK, AGC, Kleinhirn, Basalganglien } \\
\text { Depressive Symptomatik korreliert mit rCBF-Anstieg im HPK und } \\
\text { vermindert sich mit rCBF-Anstieg im DLPFK und supraorbitalem } \\
\text { präfrontalen Kortex }\end{array}$ \\
\hline \multicolumn{4}{|l|}{ Therapieeffekte } \\
\hline Kennedy et al. (2001) (15) & $\begin{array}{l}\text { Patienten }(n=13) \\
\text { Gesunde }(n=24)\end{array}$ & FDG-18F-PET & $\begin{array}{l}\text { Normalisierung des Metabolismus im präfrontalen Kortex } \\
\text { und AGC unter Paroxetin-Therapie }\end{array}$ \\
\hline Holthoff et al. (2004) (12) & Patienten $(n=41)$ & FDG-18F-PET & $\begin{array}{l}\text { Verminderung des Metabolismus im linken präfrontalen, ante- } \\
\text { rioren temporalen Kortex und AGC sowie bilateral im Bereich der } \\
\text { Basalganglien unter Citalopram bzw. Mirtazapin-Therapie }\end{array}$ \\
\hline \multicolumn{4}{|l|}{ Responderanalysen } \\
\hline Mayberg et al. (2000) (20) & Patienten $(n=17)$ & FDG-18F-PET & $\begin{array}{l}\text { Verminderung des Metabolismus im subgenualen AGC, HPK, } \\
\text { Insula, verstärkter Metabolismus im präfrontalen und parietalen } \\
\text { Kortex, AGC nach 6wöchiger Therapie bei den Respondern unter } \\
\text { Paroxetin-Therapie }\end{array}$ \\
\hline Little et al. (2005) (19) & $\begin{array}{l}\text { Patienten }(n=20) \\
\text { Gesunde }(n=20)\end{array}$ & FDG-18F-PET & $\begin{array}{l}\text { Responder auf Bupropion bzw. Venlafaxin-Therapie zeigten einen } \\
\text { frontalen und temporalen Hypometabolismus }\end{array}$ \\
\hline
\end{tabular}

matik bzw. eine Normalisierung der Hypofrontalität mit der Therapieresponse. In letzter Zeit zeigte sich die funktionelle Charakterisierung des rostralen anterioren Gyrus cinguli, der als Schnittstelle zwischen Emotion, Kognition und Verhalten fungiert, als besonders geeignet, Therapiewirkungen im Verlauf zu beobachten und Therapie-Responder von Nicht-Respondern zu differenzieren (ausgewählte Literatur s. Tab. 1).

\section{Experimentelle Traurigkeit}

Mit Hilfe der Positronen-Emissions-Tomographie untersuchten Liotti und Kollegen Zustände von Angst, Traurigkeit bzw. emotionaler Gleichgültigkeit, die sie mit Hilfe von autobiographischen Gedächtnisprotokollen induzierten (17). Es zeigte sich, dass bei der Konfrontation mit den Gedächtnisprotokollen Angst und Traurigkeit zu spezifischen Aktivierungsmustern in neokortikalen und limbischen Regionen führen, die auch unter BaselineBedingungen bei der depressiven
Störung Veränderungen aufweisen. Traurigkeit ging dabei mit einer selektiven dorsalen neokortikalen Aktivitätsabnahme einher, während Angst mit einer Aktivitätszunahme ventraler Hirnareale vergesellschaftet war. Die gleiche Arbeitsgruppe wies außerdem neokortikale Deaktivierungen und verstärkte limbisch-paralimbische Aktivierungen nach (21). Das Erleben von Traurigkeit scheint insgesamt mit einer Aktivitätszunahme evolutionsgeschichtlich alter, limbischer Areale des Gehirns assoziiert zu sein. Vorübergehend „heruntergefahren“ werden bei Traurigkeit hingegen evolutionär jüngere Regionen des Gehirns, die funktionell bei höheren kognitiven Funktionen (Aufmerksamkeit, Bewertung, Einordnung ins Ganze) beteiligt sind.

\section{Studien mit Aktivierungs- paradigmen}

Bei der Untersuchung höherer kognitiver Funktionen mit Hilfe von Aktivierungsparadigmen (z.B. bei
Denkaufgaben wie dem „Tower-ofLondon“-Problem) lassen sich in Übereinstimmung mit dem Netzwerk der induzierten Traurigkeit sowie den funktionellen Ruhebefunden Hinweise auf eine gestörte Funktion frontaler Areale, des rostralen Gyrus cinguli sowie der Basalganglien bei der depressiven Störung finden (ausgewählte Literatur s. Tab. 2). Es zeigte sich, dass depressive Patienten im Gegensatz zu gesunden Probanden nur sehr begrenzt über den anterioren Gyrus cinguli verfügen können (23). Dabei lassen Befunde über eine mediofrontale und cinguläre Hyperaktivität bei der Verarbeitung trauriger Bilder eher eine funktionelle Dysregulation statt einer durchgehenden Hypoaktivität dieser Areale wahrscheinlich werden. Diese Annahme wird gestützt durch eine fMRT-Untersuchung, bei der zu Beginn der depressiven Erkrankung und nach Remission Bilder mit unterschiedlicher affektiver Valenz präsentiert wurden. Es ließ sich bei den Patien- 
Tab. 2 Aktuelle Untersuchungen mit Aktivierungsparadigmen

\begin{tabular}{|c|c|c|c|c|}
\hline Autoren & Stichprobe & $\begin{array}{l}\text { Untersuchungs- } \\
\text { methode }\end{array}$ & Paradigma & Hauptbefunde \\
\hline Surguladze et al. (2005) (28) & $\begin{array}{l}\text { Patienten }(n=16) \\
\text { Gesunde }(n=14)\end{array}$ & fMRT & $\begin{array}{l}\text { Visuelle Präsentation } \\
\text { fröhlicher und } \\
\text { trauriger Gesichter }\end{array}$ & $\begin{array}{l}\text { Patienten zeigten linearen Aktivierungs- } \\
\text { anstieg im Bereich der Basalganglien, } \\
\text { der AMY/HPK auf steigende Intensität der } \\
\text { dargestellten Traurigkeit }\end{array}$ \\
\hline Fu et al. (2004) (10) & $\begin{array}{l}\text { Patienten }(n=19) \\
\text { Gesunde }(n=19)\end{array}$ & fMRT & $\begin{array}{l}\text { Visuelle Präsentation } \\
\text { trauriger Gesichter }\end{array}$ & $\begin{array}{l}\text { Reduzierte Aktivität der li. AMY, des } \\
\text { ventralen Striatums und des fronto- } \\
\text { parietalen Kortex, unter Symptomverbes- } \\
\text { serung veränderte Aktivität im Bereich } \\
\text { des prägenualen AGC }\end{array}$ \\
\hline Lawrence et al. (2004) (16) & $\begin{array}{l}\text { Patienten }(n=21) \\
\text { Gesunde }(n=11)\end{array}$ & fMRT & $\begin{array}{l}\text { Visuelle Präsentation } \\
\text { von Gesichtern unter- } \\
\text { schiedlicher emotio- } \\
\text { naler Valenz }\end{array}$ & $\begin{array}{l}\text { Schwere der Symptomatik korrelierte mit } \\
\text { Reaktion des HPK auf traurige Gesichter, } \\
\text { Pat. mit majorer Depression zeigten an- } \\
\text { deres Aktivierungsverhalten als bipolare } \\
\text { Patienten (verminderte Aktivierung) }\end{array}$ \\
\hline Davidson et al. (2003) (5) & $\begin{array}{l}\text { Patienten }(n=12) \\
\text { Gesunde }(n=5)\end{array}$ & fMRT & $\begin{array}{l}\text { Visuelle Präsentation } \\
\text { emotional unter- } \\
\text { schiedlich valenten } \\
\text { Bildmaterials }\end{array}$ & $\begin{array}{l}\text { Patienten mit höherer Reaktion des AGC } \\
\text { auf negative Stimuli zu Beginn der Be- } \\
\text { handlung zeigten den größten Therapie- } \\
\text { erfolg }\end{array}$ \\
\hline Elliot et al. (2002) (7) & $\begin{array}{l}\text { Patienten }(n=10) \\
\text { Gesunde }(n=11)\end{array}$ & fMRT & $\begin{array}{l}\text { Go/NoGo-Task mit } \\
\text { emotional unter- } \\
\text { schiedlich valenten } \\
\text { Wörtern }\end{array}$ & $\begin{array}{l}\text { Patienten zeigten verminderte Reaktion } \\
\text { auf emotionale Wörter im ventralen ACG } \\
\text { und posterioren OFC und vermehrte } \\
\text { Aktivierung auf traurige Reize im rostra- } \\
\text { len ACG bis zum anterioren medialen } \\
\text { präfrontalen Kortex }\end{array}$ \\
\hline
\end{tabular}

ten zu Beginn der Untersuchung eine gestörte Verarbeitung des affektiv negativen Stimulusmaterials im Bereich der linken Inselregion, links präfrontal sowie im linken anterioren Gyrus cinguli nachweisen, die sich unter antidepressiver Medikation mit Venlafaxin zum Teil wieder normalisierte (5).

Auch bei Patienten mit einer depressiven Störung wurde die Methode der Emotionsinduktion durch autobiographische Gedächtnisprotokolle angewandt. Hierbei zeigten die depressiven Patienten bei der Induktion trauriger Emotionen ein abweichendes Aktivierungsverhalten in den basalen und lateralen Anteilen des Frontallappens. Bei diesen führten die traurigen Erinnerungen zu einer Aktivitätsminderung orbitofrontaler Areale, während bei Gesunden eine vermehrte Aktivierung des subgenualen Gyrus cinguli und eine verminderte Aktivierung dorsolateralpräfrontaler Areale zu beobachten war. Dieses unterschiedliche Aktivierungsverhalten war sowohl bei den akut erkrankten als auch bei den re- mittierten Patienten nachzuweisen, so dass es als Zeichen einer anhaltenden Veränderung emotionaler Regelkreise - gewissermaßen als TraitMarker - diskutiert wird (18).

Eine Schlüsselregion im Zusammenhang mit der emotionalen Dysregulation bei depressiven Störungen ist auch die Amygdala-Formation. Es wurde gezeigt, dass bei maskierter, also bei unbewusst bleibender Präsentation ängstlicher Gesichter die Reagibilität der Amygdala bei depressiven Patienten deutlich stärker ist und dass sich dieser Befund unter einer antidepressiven Behandlung wieder normalisiert (25). Zudem wurde bei depressiven Patienten eine zeitlich verlängerte Antwort der Amygdala auf emotional negative Reize beschrieben. Dies könnte ein Korrelat depressiver Grübelneigung sein (26). Außerdem ist seit langem ein Zusammenhang der Amygdalareagibilität mit dem Serotonin-Transportergen im Sinne eines Vulnerabilitätsfaktors für Angst und Depression beschrieben.

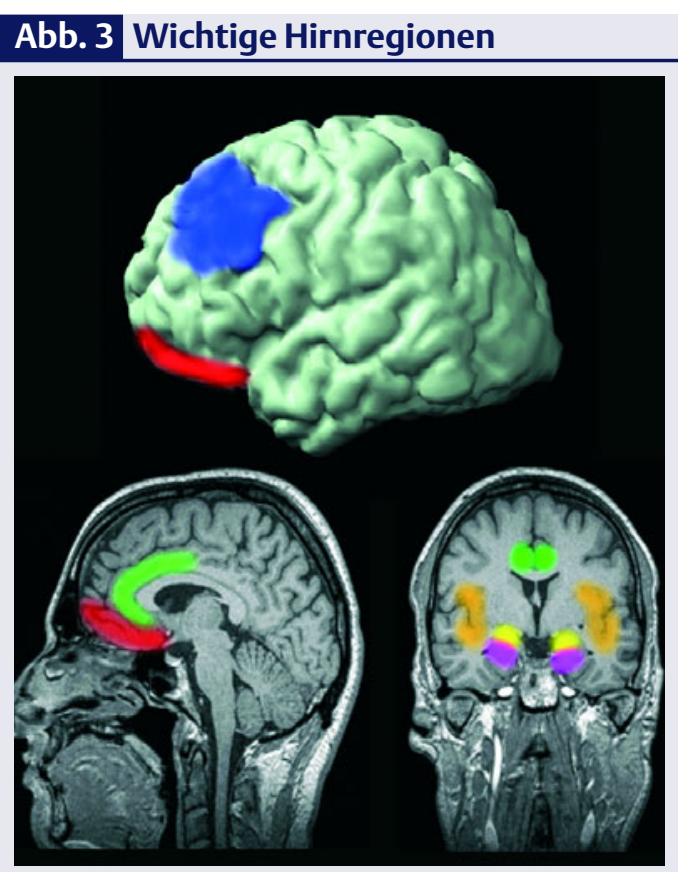

Wichtige Hirnregionen, die bei depressiven Störungen funktionelle Veränderungen aufweisen. Gelb: Amygdala; Violett: Hippokampus; Orange: Insula; Grün: anteriorer Gyrus cinguli; Rot: orbitofrontaler präfrontaler Kortex; Blau: dorsolateraler präfrontaler Kortex (nach 32) 


\section{Depression als Netzwerk- störung}

Versucht man nun die dargestellten Befunde zu integrieren, so wird deutlich, dass sie auf solche Hirnareale fokussieren, die auch beim Gesunden an der Verarbeitung von Emotionen beteiligt sind. Diese Hirnregionen lassen sich in einem Modell der Depression als Netzwerkstörung vereinen. Beim Gesunden geht dabei affektive Modulation mit der geordneten Interaktion evolutionär jüngerer dorsal-neokortikaler und älterer, ventral-limbischer Hirnregionen einher (dorsal: dorsolateral-präfrontaler Kortex, dorsaler anteriorer Gyrus cinguli, posteriorer Parietallappen bzw. ventral: Hippokampus-Amygdala-Formation, subgenualer Gyrus cinguli, anteriore Inselregion, Hypothalamus, s. Abb. 3). In Bezug auf die Herausbildung physiologischer als auch pathologischer emotionaler Zustände kommt dem subgenualen anterioren Gyrus cinguli eine wichtige Funktion zu, dort wird wohl zwischen den „ventralen“ Impulsen und der Hemmung durch das „dorsale“ Netzwerk regulatorisch vermittelt.

Trotz einiger stabiler Ergebnisse ist die Studienlage durch eine gewisse Heterogenität und zum Teil bestehende Widersprüchlichkeit gekennzeichnet. Dies ist zum einen begründet in methodischen Einschränkungen und Mängeln, aber auch in der enormen klinischen Bandbreite der Störungen, die sich unter dem Oberbegriff der „Depression“ zusammenfassen lassen. Diese Heterogenität aufzuklären kann Aufgabe zukünftiger Bildgebungsstudien unter Berücksichtigung von Vulnerabilitätsgenen (11) und der Gen-Umweltinteraktion (4) sein.

\section{Fazit}

Unter Berücksichtigung einiger Limitierungen haben bildgebende Verfahren die Erforschung der Pathophysiologie der Depression auf der Systemebene in den letzten Jahren nachhaltig bereichert. Dabei zeigten unterschiedliche Studien, dass eine therapeutisch bewirkte Symptomverbesserung mit einer Normalisierung kortikaler und limbischer Aktivierungsmuster bzw. ei- ner Normalisierung metabolischer Parameter vergesellschaftet ist. Weiterhin lässt das Aktivierungsverhalten funktioneller Schlüsselregionen wie dem subgenualen anterioren Cingulum, limbischer und dorsalpräfrontaler kortikaler Areale Rückschlüsse auf die Therapie-Response zu. Zukünftig werden bildgebende Verfahren in Kombination mit Molekularbiologie, experimenteller Affektpsychologie und Neuropsychologie über Netzwerkmodelle und über das Identifizieren valider neurofunktioneller Subgruppen bei der Aufklärung der biologischen Heterogenität affektiver Störungen und der Evaluation therapeutischer Interventionen entscheidend beteiligt sein, was in der Folge effizientere, individuell zugeschnittene Behandlungsstrategien ermöglichen soll.

\section{Depression and neuroimaging}

Modern neuroimaging has effectively advanced the implementation of network models into research of depressive disorders. Distinct morphological findings report alternations in brain regions, which are part of the emotional network. Functional neuroimaging points to disturbances of regions like frontal cortex, limbic system involving the hippocampus-amygdalaformation and finally the basal ganglia. Affective modulation depends on an ordered interaction of ventral-limbic and dorsal-neocortical regions of the brain, which become unbalanced in depressive disorders, whereas the rostral part of the anterior cingulate gyrus seems to be a key region. In the future neuroimaging research will focus on the identification of valid neurofunctional subgroups based on this model having regard to vulnerability genes. This will encourage the development of more efficient therapy strategies.

\section{Key Words}

affective disorders - neuroimaging PET/Spect - MRI - MR-spectroscopy

\section{Literatur}

1. Abercrombie HC, Schaefer SM, Larson $\mathrm{CL}$, Oakes TR, Lindgren KA, Holden JE, Perlman SB, Turski PA, Krahn DD, Benca RM, Davidson RJ. Metabolic rate in the right amygdala predicts negative affect in depressed patients. Neuroreport 1998; 9(14): 3301-3307
2. Ahearn EP, Jamison KR, Steffens DC, Cassidy F, Provenzale JM, Lehman A, Weisler RH, Carroll BJ, Krishnan KR. MRI correlates of suicide attempt history in unipolar depression. Biol Psychiatry 2001; 50(4): 266-270 3. Bremner JD, Innis RB, Salomon RM, Staib LH, Ng CK, Miller HL, Bronen RA, Krystal JH, Duncan J, Rich D, Price LH, Malison R, Dey H, Soufer R, Charney DS. Positron emission tomography measurement of cerebral metabolic correlates of tryptophan depletion-induced depressive relapse. Arch Gen Psychiatry 1997; 54(4): 364-374

4. Caspi A, Sugden K, Moffitt TE, Taylor A, Craig IW, Harrington H, McClay ], Mill ], Martin J, Braithwaite A, Poulton R. Influence of life stress on depression: moderation by a polymorphism in the 5 -HTT gene. Science 2003; 301(5631): 386-389

5. Davidson RJ, Irwin W, Anderle MJ, Kalin $\mathrm{NH}$. The neural substrates of affective processing in depressed patients treated with venlafaxine. Am J Psychiatry 2003; 160(1): 64-75

6. Drevets WC, Price JL, Simpson JR, Jr., Todd RD, Reich T, Vannier M, Raichle ME. Subgenual prefrontal cortex abnormalities in mood disorders. Nature 1997; 386(6627): 824-827

7. Elliott R, Rubinsztein SS, Sahakian B], Dolan RJ. The neural basis of mood-congruent processing biases in depression. Arch Gen Psychiatry 2002; 59(7): 597-604

8. Ende G, Braus DF, Walter S, Weber-Fahr $W$, Henn FA. The hippocampus in patients treated with electroconvulsive therapy: a proton magnetic resonance spectroscopic imaging study. Arch Gen Psychiatry 2000; 57(10): 937-943

9. Frodl T, Meisenzahl EM, Zill P, Baghai T, Rujescu D, Leinsinger $G$, Bottlender R, Schule C, Zwanzger P, Engel RR, Rupprecht $\mathrm{R}$, Bondy B, Reiser M, Moller HJ. Reduced hippocampal volumes associated with the long variant of the serotonin transporter polymorphism in major depression. Arch Gen Psychiatry 2004; 61(2): 177-183

10. Fu CH, Williams SC, Cleare AJ, Brammer M], Walsh ND, Kim J, Andrew CM, Pich EM, Williams PM, Reed LJ, Mitterschiffthaler MT, Suckling J, Bullmore ET. Attenuation of the neural response to sad faces in major depression by antidepressant treatment: a prospective, event-related functional magnetic resonance imaging study. Arch Gen Psychiatry 2004; 61(9): 877-889

11. Heinz A, Braus DF, Smolka MN, Wrase J, Puls I, Hermann D, Klein S, Grusser SM, Flor H, Schumann G, Mann K, Buchel C. Amygdala-prefrontal coupling depends on a genetic variation of the serotonin transporter. Nat Neurosci 2005; 8(1): 20-21

12. Holthoff VA, Beuthien-Baumann B, Zundorf G, Triemer A, Ludecke S, Winiecki P, Koch R, Fuchtner F, Herholz K. Changes in brain metabolism associated with remission in unipolar major depression. Acta Psychiatr Scand 2004; 110(3): 184-194

13. Kaufman MJ, Henry ME, Frederick B, Hennen J, Villafuerte RA, Stoddard EP, Schmidt ME, Cohen BM, Renshaw PF. Selective serotonin reuptake inhibitor discontinuation syndrome is associated with a 
rostral anterior cingulate choline metabolite decrease: a proton magnetic resonance spectroscopic imaging study. Biol Psychiatry 2003; 54(5): 534-539

14. Kegeles LS, Malone KM, Slifstein M, Ellis SP, Xanthopoulos E, Keilp JG, Campbell C, Oquendo M, Van Heertum RL, Mann JJ. Response of cortical metabolic deficits to serotonergic challenge in familial mood disorders. Am J Psychiatry 2003; 160(1): 76-82 15. Kennedy SH, Evans KR, Kruger S, Mayberg HS, Meyer JH, McCann S, Arifuzzman Al, Houle S, Vaccarino FJ. Changes in regional brain glucose metabolism measured with positron emission tomography after paroxetine treatment of major depression. Am J Psychiatry 2001; 158(6): 899-905

16. Lawrence NS, Williams AM, Surguladze S, Giampietro V, Brammer M], Andrew C, Frangou S, Ecker C, Phillips ML. Subcortical and ventral prefrontal cortical neural responses to facial expressions distinguish patients with bipolar disorder and major depression. Biol Psychiatry 2004; 55(6): 578587

17. Liotti M, Mayberg HS, Brannan SK, McGinnis S, Jerabek P, Fox PT. Differential limbic-cortical correlates of sadness and anxiety in healthy subjects: implications for affective disorders. Biol Psychiatry 2000; 48(1): 30-42

18. Liotti M, Mayberg HS, McGinnis S, Brannan SL, Jerabek P. Unmasking diseasespecific cerebral blood flow abnormalities: mood challenge in patients with remitted unipolar depression. Am J Psychiatry 2002; 159(11): 1830-1840

19. Little JT, Ketter TA, Kimbrell TA, Dunn RT, Benson BE, Willis MW, Luckenbaugh DA, Post RM. Bupropion and venlafaxine responders differ in pretreatment regional cerebral metabolism in unipolar depression. Biol Psychiatry 2005; 57(3): 220-228

20. Mayberg HS, Brannan SK, Tekell JL, Silva JA, Mahurin RK, McGinnis S, Jerabek PA. Regional metabolic effects of fluoxetine in major depression: serial changes and relationship to clinical response. Biol Psychiatry 2000; 48(8): 830-843

21. Mayberg HS, Liotti M, Brannan SK, McGinnis S, Mahurin RK, Jerabek PA, Silva JA, Tekell JL, Martin CC, Lancaster JL, Fox PT. Reciprocal limbic-cortical function and negative mood: converging PET findings in depression and normal sadness. Am J Psychiatry 1999; 156(5): 675-682

22. Obergriesser T, Ende G, Braus DF, Henn FA. Long-term follow-up of magnetic resonance-detectable choline signal changes in the hippocampus of patients treated with electroconvulsive therapy. J Clin Psychiatry 2003; 64(7): 775-780

23. Okada G, Okamoto Y, Morinobu S, Yamawaki S, Yokota N. Attenuated left prefrontal activation during a verbal fluency task in patients with depression. Neuropsychobiology 2003; 47(1): 21-26

24. Renshaw PF, Lafer B, Babb SM, Fava M, Stoll AL, Christensen JD, Moore CM, Yurgelun-Todd DA, Bonello CM, Pillay SS, Rothschild AJ, Nierenberg AA, Rosenbaum JF, Cohen BM. Basal ganglia choline levels in depression and response to fluoxetine treat- ment: an in vivo proton magnetic resonance spectroscopy study. Biol Psychiatry 1997; 41(8): 837-843

25. Sheline YI, Barch DM, Donnelly JM, OIlinger JM, Snyder AZ, Mintun MA. Increased amygdala response to masked emotional faces in depressed subjects resolves with antidepressant treatment: an fMRI study. Biol Psychiatry 2001; 50(9): 651-658

26. Siegle GJ, Steinhauer SR, Thase ME, Stenger VA, Carter CS. Can't shake that feeling: event-related $\mathrm{FMRI}$ assessment of sustained amygdala activity in response to emotional information in depressed individuals. Biol Psychiatry 2002; 51(9): 693-707 27. Steingard R, Renshaw P, Hennen J, Lenox M, Cintron C, Young A, Connor D, Au T, Yurgelun-Todd D. Smaller frontal lobe white matter volumes in depressed adolescents. Biol Psychiatry 2002; 52(5): 413

28. Surguladze $S$, Brammer M], Keedwell $P$, Giampietro V, Young AW, Travis MJ, Williams SC, Phillips ML. A differential pattern of neural response toward sad versus happy facial expressions in major depressive disorder. Biol Psychiatry 2005; 57(3): 201-209

29. Thomas AJ, O'Brien JT, Davis S, Ballard C, Barber R, Kalaria RN, Perry RH. Ischemic basis for deep white matter hyperintensities in major depression: a neuropathological study. Arch Gen Psychiatry 2002; 59(9): 785-792

30. Videbech P, Ravnkilde B, Pedersen TH, Hartvig $\mathrm{H}$, Egander A, Clemmensen K, Rasmussen NA, Andersen F, Gjedde A, Rosenberg $R$. The Danish PET/depression project: clinical symptoms and cerebral blood flow. A regions-of-interest analysis. Acta Psychiatr Scand 2002; 106(1): 35-44

31. Videbech P, Ravnkilde B. Hippocampal volume and depression: a meta-analysis of MRI studies. Am J Psychiatry 2004; 161(11): 1957-1966

32. Vollmert C, Tost $\mathrm{H}$, Brassen S, Jatzko A, Braus DF. Depression und moderne Bildgebung. Fortschr Neurol Psychiatr 2004; 72(8): 435-445

\section{Korrespondenzadresse}

Dr. Christian Vollmert

Prof. Dr. Dieter F. Braus

Universitätsklinikum Hamburg-Eppendorf Martinistraße 52

20246 Hamburg

cvollmert@uke.uni-hamburg.de

\section{Jetzt abonnieren!}

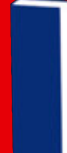

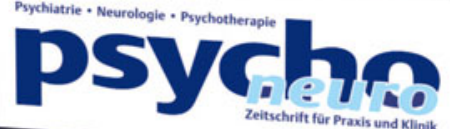

- 1

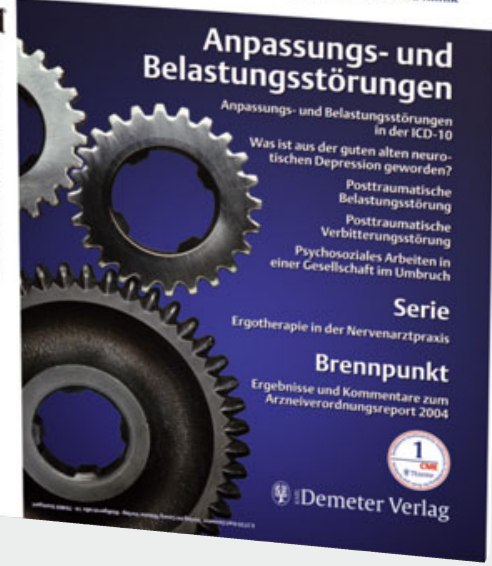

\section{Mit uns behalten Sie den Überblick}

Psychiatrie Neurologie Psychotherapie

- Übersichten /Originalarbeiten und Kasuistiken

- Schwerpunktberichte / Brennpunktberichte

- Kurze prägnante Artikel, die zum Lesen einladen

- Kompetente und zeitnahe Berichte von

Kongressen und Symposien

Neu:

- Jetzt durch die Landesärztekammer Baden-

Württemberg CME zertifiziert

-Vollständig im Internet abrufbar

Psychoneuro ist Organ der Deutschen Gesellschaft für Bipolare Störungen e.V.

Inklusive Online-Zugang*

Recherche im Volltext

www.thieme-connect.de

www.thieme.de/psychoneuro

*gilt nur für das persönliche Abonnement

Interessiert an einem Abo?

0711/8931-906

0711/8931-901

kundenservice@thieme.de

www.thieme.de

VY33 